\title{
Implicit learning of a repeated segment in continuous tracking: A reappraisal
}

\author{
Stephanie Chambaron, Dominique Ginhac, Carole Ferrel-Chapus, and Pierre Perruchet \\ Université de Bourgogne, Dijon, France
}

\begin{abstract}
Several prior studies (e.g., Shea, Wulf, Whitacre, \& Park, 2001; Wulf \& Schmidt, 1997) have apparently demonstrated implicit learning of a repeated segment in continuous-tracking tasks. In two conceptual replications of these studies, we failed to reproduce the original findings. However, these findings were reproduced in a third experiment, in which we used the same repeated segment as that used in the Wulf et al. studies. Analyses of the velocity and the acceleration of the target suggests that this repeated segment could be easier to track than the random segments serving as control, accounting for the results of Wulf and collaborators. Overall these experiments suggest that learning a repeated segment in continuous-tracking tasks may be much more difficult than learning from a repeated sequence in conventional serial reaction time tasks. A possible explanation for this difference is outlined.
\end{abstract}

Implicit learning is usually defined as the process whereby people learn without intent and without being able to clearly articulate what they learn (for a brief overview, see Cleeremans, Destrebecqz, \& Boyer, 1998). One of the more common paradigms of implicit learning is the serial reaction time (SRT) paradigm. In SRT situations, a target stimulus appears in successive trials at one of a few possible positions, and participants are asked to react to the appearance of the target by pressing a key on the keyboard. In fact, the same sequence of trials is repeated throughout the sessions. After some amount of practice, performance of participants on the repeated sequence is generally better than performance on a new sequence. In recent years, a huge number of studies using SRT tasks have improved our knowledge about various issues, such as the implicit-learning abilities of children (e.g., Meulemans, Van Der Linden, \& Perruchet, 1998), elderly people (e.g., Curran, 1997), and neurologically impaired patients (e.g., Smith, Siegert, McDowall, \& Abernethy, 2001), the relationships between performance and explicit knowledge (e.g., Shanks \& Perruchet, 2002), and the relationships between perceptual and motor components of learning (e.g., Kelly, Burton, Riedel, \& Lynch, 2003).

A far smaller number of studies involve continuous-tracking tasks (Pew, 1974, Exp. 1; Shea et al., 2001; Wulf \& Schmidt, 1997). In Pew and in Wulf and Schmidt, participants were

Correspondence should be addressed to Stephanie Chambaron, LEAD/CNRS, Université de Bourgogne, Esplanade Erasme, 21000 Dijon, France. Email: sginhac@u-bourgogne.fr

This work was supported by the Centre National de la Recherche Scientifique (CNRS, UMR 5022 and UMR 5158), the Université de Bourgogne (LEAD and LE2I), and the Région de Bourgogne (AAFE). The authors thank Charles H. Shea and two anonymous reviewers for their valuable suggestions and comments. 
asked to track a moving target by acting on a handdriven lever. The target moved along a horizontal axis, according to the $y$-value of a polynomial function. The experimental sessions consisted of a succession of trials, and, unbeknownst to participants, each trial was divided into three segments. Typically, the first and the third segments were generated by a function in which the coefficients were randomly drawn on each occasion, hence generating pseudorandom target displacements. The same function served to generate the second segment, but the coefficients were now fixed, and hence the movement described by the target around the middle of each trial was the same across the whole training session (in another condition, the repeated segment was the third one). Participants' tracking accuracy was found to be better on the repeated segment than on the pseudorandom segments. Shea et al. generalized those results to a situation in which participants had to track the target by moving the platform of a stabilometer on which they were standing.

The present study was initially intended to examine the possibility of reproducing certain results from the SRT literature in continuoustracking tasks. However, in two conceptual replications of the Wulf and collaborators' studies (Experiments 1a and 1b) we observed that tracking was no more accurate for the repeated segment than for the pseudorandom segments. Experiment 2 suggested that the positive results reported by Wulf and collaborators were in fact due to a peculiarity of their experimental procedure. The apparent difficulty of obtaining evidence of learning in continuous-tracking tasks is challenging, because it contrasts with the easy development of implicit learning in the formally similar SRT tasks. A possible reason for this discrepancy is outlined in the General Discussion.

\section{EXPERIMENT 1A}

Experiments 1a and $1 \mathrm{~b}$ shared most of the features that were common to all the studies by Wulf and collaborators. Participants had to track a target moving horizontally on the computer screen.
Each trial was divided into three segments, with the middle segment being repeated throughout the session, whereas the first and the last segments were randomly generated. Segments were generated by the same $\sin -\cos$ function as that used in most of Wulf and collaborators' studies. Other aspects of Wulf and collaborators' studies were varied between experiments, such as the specific manipulandum, the time parameters of target displacements, and the number of training trials. We did not attempt to replicate a specific experiment. In the following experiments, participants used a standard computer mouse to track the target, and several time parameters and training lengths were explored.

\section{Method}

\section{Participants}

A total of 18 first-year psychology students (15 females and 3 males) at the University of Bourgogne, France, served as participants. All of them had normal or corrected-to-normal vision, and none had prior experience with the task or were informed about the purpose of the experiment.

\section{Apparatus}

Stimulus presentation, timing, and data collection were all implemented on a PC laptop equipped with 14-in. TFT colour screen, set at a resolution of $1,024 \times 768$ pixels. The participants were seated in front of the screen at a typical viewing distance of about $65 \mathrm{~cm}$. A C ++ program computed in real time the target movements at a frequency of $200 \mathrm{~Hz}$ and displayed the target on the screen. The target was a 20-pixel blue dot moving horizontally in the middle of the computer screen. The participants used a standard mouse to track the target, and only the horizontal displacements of the mouse pointer (a 10-pixel black dot) were allowed. The mouse was calibrated such that $1 \mathrm{~cm}$ of mouse movement on the pad produced exactly $6 \mathrm{~cm}$ of movement on the screen. The mouse pointer location was recorded at a $200-\mathrm{Hz}$ frequency for use in the subsequent analyses.

\section{THE QUARTERLY JOURNAL OF EXPERIMENTAL PSYCHOLOGY, 2006, 59 (5)}




\section{Stimuli}

Target patterns were composed of three segments of equal duration, each segment being generated by a sine-cosine series of the general form:

$$
\begin{aligned}
\alpha_{i}= & b_{0}+a_{1} \sin \theta_{i}+b_{1} \cos \theta_{i}+a_{2} \sin 2 \theta_{i} \\
& +b_{2} \cos 2 \theta_{i}+a_{3} \sin 3 \theta_{i}+b_{3} \cos 3 \theta_{i} \\
& +a_{4} \sin 4 \theta_{i}+b_{4} \cos 4 \theta_{i}+a_{5} \sin 5 \theta_{i} \\
& +b_{5} \cos 5 \theta_{i}+a_{6} \sin 6 \theta_{i}+b_{6} \cos 6 \theta_{i}
\end{aligned}
$$

where $\theta_{i}=1.5 \pi(i+ø) /\left(\right.$ freq* $q^{*}$ time $)$. Freq was the sampling frequency (i.e., $200 \mathrm{~Hz}$ ), time was the segment period (i.e., $12 \mathrm{~s}$ ), and $\varnothing$ was the phase shift, which allowed the segment to start and/or end at any predetermined position. The $\alpha_{i}$ value represented horizontal localization of the target on the computer screen. Note that only a part of the whole period ( $1.5 \pi$ instead of $2 \pi$ ) was used, in order to make the target position at the end of a segment independent from its starting position.

Each of the three segments (S1, S2, and S3) had its own coefficients. The only difference between the three segments was that the coefficients for S1 and S3 differed for each trial, whereas the coefficients for S2 were constant over trials for any given participant. The coefficients $(\mathrm{a} 1 \rightarrow \mathrm{a} 6$ and $\mathrm{b} 1 \rightarrow \mathrm{b} 6)$ for the three segments were computed for each participant before the experiment began, with the following two criteria: (a) coefficients were numbers within the range of +5 to -5 , and (b) there was no more than a $10 \%$ difference between the maxima (or minima) of the curves of the three segments. This latter criterion was used to verify that no large changes of target magnitude occurred between S1, S2, and S3. The phase shift ( $\varnothing)$ and $b_{0}$ were set in such a way that (a) the beginning and the end of the segments were randomly located on the horizontal axis, and (b) the slopes at the beginning and at the end of the S2 segment were equal to zero, which implied a change in target direction at the S2 boundaries.

\section{Procedure}

Participants were told that they would see a blue dot (the target) moving horizontally on the screen and that their task consisted in tracking this dot with the mouse pointer. They were asked to put the pointer on the centre of the dot as precisely as possible. They were not informed about the presence of a repeated segment.

The training phase consisted of 12 trials of $36 \mathrm{~s}$ each, separated by a break of about $10 \mathrm{~s}$. The first (S1) and the third (S3) segments changed on every trial. None of the random segments was repeated across the experiment. In contrast, the middle segment (S2) was identical from trial to trial for any given participant, although this segment differed for each participant.

\section{Results}

The dependent variable was the root mean square error ( $R M S E$, in screen pixels) between the centre of the mouse pointer and the centre of the target. An analysis of variance (ANOVA) was performed on the RMSE with trials (12) and segments (repeated vs. random) as within-subject factors. The RMSE for the repeated segment was computed on $\mathrm{S} 2$, whereas the RMSE for the random segments were computed on S1 and S3. As shown in Figure 1a, there was a striking improvement in performance, which elicited a significant main effect of trials, $F(11,187)=12.33, p<$ .0001 . However, neither the main effect of segments, $F(1,17)=0.08, p=.78$, nor the trials by segment interaction, $F(11,187)=0.49, p=.91$, reached significance. These results show that even though participants' performance improved across training, they did not benefit from the repetition structure.

\section{EXPERIMENT 1B}

Detecting the repetition of a segment can have a beneficial effect on tracking only if anticipating the movement of the target allows some improvement in tracking accuracy. If the task is very easy to perform from the outset, then any further 

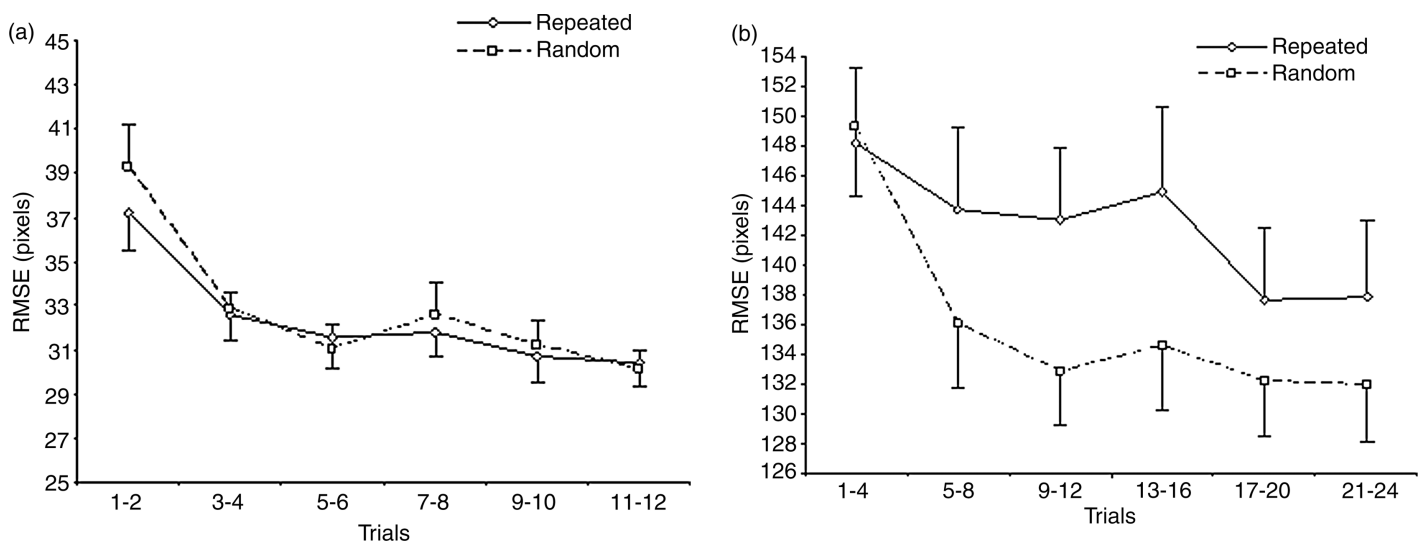

Figure 1. Evolution of RMSEs with practice for repeated and random segments in Experiments 1a and 1b. Lower RMSE scores indicate better performance. Data are averaged across sets of two trials (Figure 1a) or four trials (Figure 1b). Error bars represent standard deviations. The scale on the $\mathrm{y}$-axis has been translated to accommodate the differences observed between experiments with regard to the mean value of the RMSE (see text), but the intervals are constant, so that the differences between repeated and random segments can be directly compared.

improvement in performance due to the knowledge of the sequence may be undetectable. This situation could occur when the target is moving too slowly. The duration of a trial (given that trial duration equals the period of the $\sin -\cos$ function; the longer the trial the slower the target movement) in Wulf and collaborators' studies varied between experiments (15 s in Wulf \& Schmidt, 1997, Exp. 2; $30 \mathrm{~s}$ in Wulf \& Schmidt, Exp. 1; 75 s in Shea et al., 2001, Exps. 1 and 2). In Experiment 1a, we set the trial duration to $36 \mathrm{~s}$. Experiment $1 \mathrm{~b}$ is similar to Experiment 1a, except that the duration of a trial was shortened from $36 \mathrm{~s}$ to $15 \mathrm{~s}$, in order to increase the difficulty of tracking. In addition, the number of trials was increased from 12 to 24 .

\section{Method}

\section{Participants}

A total of 24 psychology students (19 females and 5 males) at the University of Bourgogne were recruited for the study, and none of them was familiar with the experimental task. All reported normal or corrected-to-normal vision.

\section{Apparatus, stimuli, and procedure}

The apparatus, stimuli, and procedure were identical to those used previously; except that the total duration of each trial was set to $15 \mathrm{~s}$. Participants practised the pursuit-tracking task over 24 trials in a single session.

\section{Results}

Mean RMSEs were substantially higher in Experiment $1 \mathrm{~b}$ than in Experiment 1a. Thus increasing the speed of target displacement presumably increased the difficulty of tracking in notable proportions. An ANOVA was performed on the $R M S E$ with trials $(N=24)$ and segment (repeated vs. random) as within-subjects factors. There was a significant effect of trials, $F(23,529)$ $=3.01, p<.0001$, indicating an improved performance over the trials. However, as shown in Figure $1 \mathrm{~b}$, the tracking accuracy appears to have been better for the random segment than for the repeated segment. Nevertheless, the difference did not reach significance, $F(1,23)=1.42, p=$ .2453 , and did not change reliably over the trials, as revealed by the nonsignificant trials by segment interaction, $F(23,529)=1.39, p=.1064$.

\section{Discussion of Experiments 1a and 1b}

Experiment 1a failed to reveal a selective improvement of tracking accuracy on the repeated

\section{8}

THE QUARTERLY JOURNAL OF EXPERIMENTAL PSYCHOLOGY, 2006, 59 (5) 

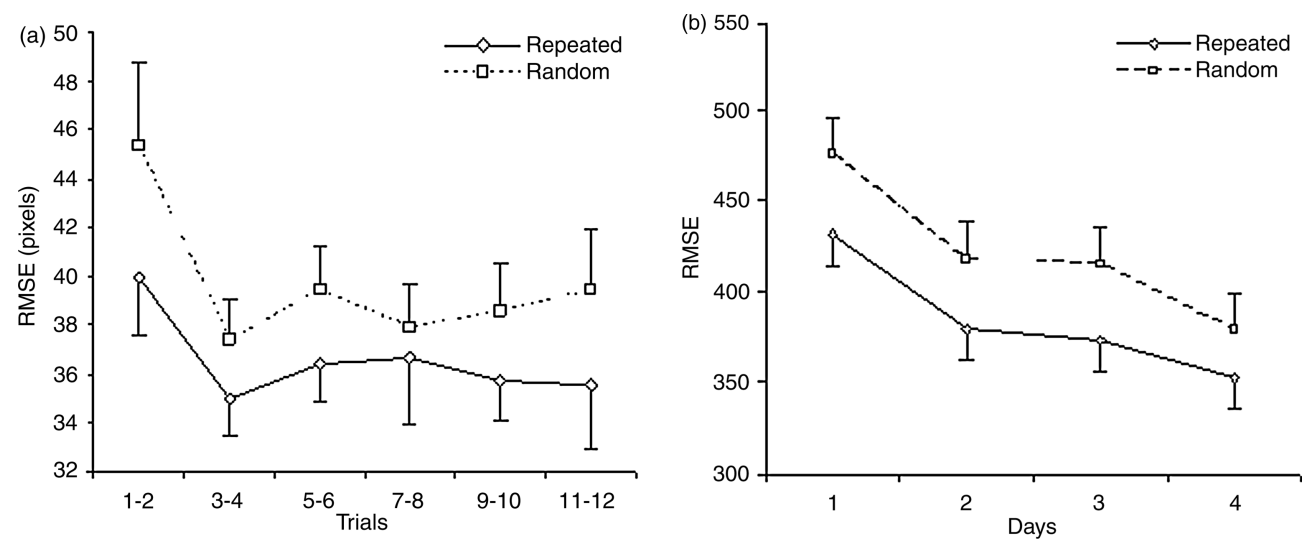

Figure 2. Evolution of RMSEs across trials for repeated and random segments in Experiment 2 (Figure 2a). Data are averaged across sets of two trials. Error bars represent standard deviations. For the purposes of comparison, the data obtained by Wulf and Schmidt with the same repeated segment (1997, Exp. 1; from their Table 1) are plotted on the right panel (Figure 2b).

segment. Increasing target velocity in Experiment $1 \mathrm{~b}$ was no more successful.

An obvious difference between ours and earlier experiments lies in the effector involved in the tasks. We used a mouse task, whereas prior studies used either a hand-driven lever (Pew, 1974; Wulf \& Schmidt, 1997) or a stabilometer (Shea et al., 2001). It is known from research on motor learning that the laws of learning may partly differ as a function of the motor system involved in the task. In particular, Wulf and Shea (2002) have emphasized that the principles derived from the study of simple skills do not always generalize to complex-skill learning. However, it is rather difficult to account for the present pattern of results along those lines. Indeed, the hand-driven lever task was designed to involve only one degree of freedom (the elbow flexion/extension), a property that defines it as a simple task in the Wulf and Shea framework. The stabilometer used by Shea et al. is the exact opposite, since its control requires whole-body movements. It is likely that the control of a mouse lies roughly in between along this dimension of complexity. Thus, although the hypothesis cannot be ruled out altogether, it appears somewhat unlikely that learning a given structure would succeed when tracking is performed through manipulanda as different as hand-driven levers and stabilometers, whereas learning the very same structure with a mouse device does not succeed.

A possible explanation for our failure to reproduce Wulf and collaborators' results lies in the smaller amount of practice received by our participants. We used 12 (Experiment 1a) and 24 (Experiment 1b) training trials in a single session, whereas Wulf and collaborators trained their participants on four days (with a number of trials per day going from 14 in Shea et al., 2001, Exps. 1 and 2, to 120 in Wulf \& Schmidt, 1997, Exp. 2). However, it is worth stressing that no improvement in performance across days is apparent in most of the Wulf and collaborators' studies (see below, discussion of Experiment 2, and Figure 2b). A further argument against this explanation comes from an exploratory experiment (Chambaron \& Ginhac, unpublished) in which the amount of practice was increased. This experiment $(N=6)$ was similar to Experiment 1a, except that participants practised the pursuittracking task over four consecutive days, with 20 trials per day. Again no reliable evidence of learning was observed. Thus the chance that our failure to reproduce previously published results could be due to an insufficient amount of practice is weak at best.

Although neither the use of a computer mouse nor the amount of training appears promising to 
explain our failure to reproduce Wulf and collaborators' findings, a number of other nonexclusive causes are possible. We noted above that the task must be complex enough for there to be a chance that anticipating the movement of the target triggers some improvement in tracking accuracy, and the velocity of the target in Experiment $1 \mathrm{~b}$ was increased with this objective. In keeping with this line of reasoning, it is possible that the movement needed to track the target in our experimental set-up was too weak in amplitude to benefit from the knowledge of the repeated sequence (the maximal movement required to track the cursor with the mouse was about $4 \mathrm{~cm}$ in length). Thus participants could have detected the repeated sequence without any detectable consequence, because anticipating the cursor displacement would be without a beneficial effect. ${ }^{1}$

Irrespective of the relevance of the abovementioned factors, another possible cause for our replication failure is the choice of the repeated segment, which differed between ours and earlier experiments. We used the same trigonometric function to generate the displacement of the target as in the studies by Wulf and collaborators. However, while we used a different set of parameters for each participant, Wulf and collaborators used a unique set for a given experiment, and, furthermore, the same set of parameters was used in several experiments (namely, Wulf and Schmidt, 1997, Exp. 1, AMP condition; Shea et al., 2001, Exps. 1 and 2). This method is questionable, because it leaves open the possibility that the repeated and the random segments differ with regard to their inherent characteristics. In most SRT studies (e.g., Shanks \& Perruchet, 2002), the choice of the repeated sequence is counterbalanced over groups, in order to ensure that any eventual difference in performance is due to repetition, rather than to the specific characteristics of the repeated sequence.

Is the repeated segment used in most of the Wulf and collaborators studies (hereafter referred to as the standard repeated segment) endowed with peculiar characteristics? It can reasonably be assumed that the difficulty of tracking partly varies as a function of target velocity and acceleration. We computed the mean velocity (i.e., the first time derivative of position) and the mean acceleration (i.e., the second time derivative of position, or the first time derivative of velocity) of the target for the standard repeated segment, and then we computed the same values for 10,000 randomly generated series, using the constraints involved in generating the segments in ours and Wulf and collaborators' experiments. Only $22 \%$ of the randomly generated segments had a mean velocity equal to or above the mean velocity of the standard repeated segment. Furthermore, only $15.95 \%$ of the randomly generated segments had a mean acceleration equal to or above the mean acceleration of the standard repeated segment. Thus if one takes target velocity and acceleration as rough measures of the ease of tracking, more than $80 \%$ of the randomly generated segments were in fact harder to track than the standard repeated segment. This suggests that at least a part of the earlier positive evidence of learning could be due to the choice of a biased repeated segment. This hypothesis was tested in the next experiment.

\section{EXPERIMENT 2}

In this experiment, we used the same standard repeated segment as that used in most of the Wulf and collaborators studies, while otherwise retaining the general procedure of Experiment 1.

\section{Method}

\section{Participants}

A total of 22 undergraduate students (18 females and 4 males) were recruited to take part in the study. Participants were unfamiliar with the

\footnotetext{
${ }^{1}$ We thank Dr. Shea for this suggestion. As a support, Dr. Shea mentioned a pilot experiment of his own in which reducing the amplitude of the target patterns (and hence of the participant's movement) cancelled out the differences in tracking accuracy between a simple sine wave and a random pattern.
} 
experimental task. All of them had normal or corrected-to-normal vision.

\section{Apparatus, stimuli, and procedure}

The same apparatus and stimuli were employed as those in Experiment 1. The procedure was identical to that of Experiment 1a, with a single training session comprising twelve 36-s long trials, each separated by a break of $10 \mathrm{~s}$. However, here the repeated segment was the same for all participants. Coefficients for this segment (the standard repeated segment) were those used in most of the Wulf and collaborators studies. They were as follows: $\mathrm{b}_{0}=-1.52 ; \mathrm{a}_{1}=-4.0 ; \mathrm{b}_{1}=3.0 ; \mathrm{a}_{2}=$ $-4.0 ; b_{2}=-3.6 ; a_{3}=3.9 ; b_{3}=4.5 ; a_{4}=0.0 ;$ $\mathrm{b}_{4}=1.0 ; \mathrm{a}_{5}=-3.8 ; \mathrm{b}_{5}=-0.5 ; \mathrm{a}_{6}=1.0 ;$ and $\mathrm{b}_{6}=2.5$.

\section{Results}

An ANOVA was performed with trial $(N=12)$ and segment (repeated vs. random) as withinsubject factors. The effect of trial was significant, $F(11,231)=3.59$. As shown in Figure 2a, performance improved over the first two trials, then remained stable across the remaining trials. More importantly, the tracking accuracy was better for the repeated segment than for the random segments. The difference was significant, $F(1,21)=$ 26.83, $p<.0001$, and the effect size was large (partial eta square $=.56$ ). However, this difference did not increase over trials, as indicated by the nonsignificant trial by segment interaction, $F(11,231)=0.59, p=.8304$.

\section{Discussion}

These results replicate to a large extent those obtained by Wulf and collaborators in their experiments using the standard repeated segment. Indeed, in these experiments, the size of the effect of segment was large, whereas the day by segment interaction was weaker or even inexistent. To illustrate, Figure $2 \mathrm{~b}$ displays the RMSE collected in Wulf and Schmidt (1997, Exp. 1) in the condition involving the standard repeated segment (the values are drawn from their
Table 1). No improvement across days is apparent. In a more general way, we computed partial eta square values as a measure of effect size on the Wulf and Schmidt (1997, Exp. 1) data, and we obtained .77 for the main effect of segment, and.08 for the interaction (in this computation, the data collected on two different repeated segments are pooled). Shea et al. (2001) did not provide the information needed to compute effects size, but it is worth noting that, although there was a significant day by segment interaction in their first experiment, their second experiment failed to obtain a similar effect, despite the fact that both experiments followed a nearly identical design (actually, as assessed from a visual inspection of Shea et al.'s Figure 4, the difference between repeated and random segments was even slightly larger for Days 1 and 2 than for Days 3 and 4). Thus, although a significant day by segments interaction is sometimes observed in Wulf and collaborators' studies, this interaction did not occur as a strong and pervasive finding.

\section{GENERAL DISCUSSION}

We first failed to obtain reliable evidence of learning in two experiments (Experiments 1a and 1b) when following the design of the Wulf and collaborators studies. In those experiments we used a different repeated segment for each participant to match, across the whole sample, the characteristics of repeated and random segments that modulate their tracking difficulty. By contrast, the same repeated segment was used for all participants in most of the Wulf and collaborators experiments. When using this segment in Experiment 2, we found a large difference between repeated and random segments.

Is it possible to infer from our study that if Wulf and collaborators had controlled for the repeated segment, they would have obtained no evidence for learning? The question is debatable given the procedural differences between ours and Wulf and collaborators' studies. We have listed in the discussion on Experiments 1a and $1 \mathrm{~b}$ a subset of those differences. Although the 
differences concerning the effectors involved in tracking and the amount of training do not seem to be good candidates to account for our failure to replicate earlier results, we outlined other features that may have prevented learning, such as the use of a nonoptimal amplitude of movement. The effect of this and other features needs to be explored in further studies. However, on the whole, we believe it to be reasonable to posit that what was taken as evidence of learning in the Wulf and collaborators' studies can be at least partly attributed to the selection of the repeated segment. Indeed, we showed that the velocity and the acceleration of the target in this segment were lower than those in the random segments used to assess the baseline. Assuming that moving a hand-lever (Wulf \& Schmidt, 1997) and controlling a stabilometer (Shea et al., 2001) would be unaffected by the velocity and the acceleration of the target looks implausible. The fact that differences between tracking accuracy for repeated and random segments were observed very early during training in most of Wulf and collaborators' studies provides an additional support for this account.

We do not claim, however, that learning from the repetition of a segment in a continuoustracking task is impossible. We do not even assert that all of the results reported by Wulf and collaborators are inconclusive. A possible exception is provided in Wulf and Schmidt (1997, Exp. 2). The mathematical properties of the series were still far from being optimal. An analysis performed as above (see Discussion of Experiments $1 \mathrm{a}$ and $1 \mathrm{~b}$ ) revealed that only $25.68 \%$ of the randomly generated segments had a mean velocity equal to or above the mean velocity of this segment, and only $37.82 \%$ of the randomly generated segments had a mean acceleration equal to or above to its mean acceleration. Thus again, the repeated segment was presumably easier to track than the randomly generated segments serving as a baseline. However, in contrast with the other experiments of the same authors and those reported above, the difference in tracking accuracy between the repeated and the random segments clearly increased across the four days of training. This pattern makes it reasonable to conclude that genuine learning may have occurred in this experiment in which, notably, the segments were generated by a simpler function than that in the other experiments (by deleting the last six terms-i.e., those generating the components of highest frequencies, from Equation 1), and the training duration was especially impressive (four days with 120 trials per day). To leave open this possibility, we adopt here the conservative conclusion according to which learning in continuous-tracking task is far more difficult to establish than the Wulf and collaborators studies would suggest. $^{2}$

A first implication of this conclusion concerns the relationship between performance improvement and the explicit knowledge of the to-belearned structure. Wulf and Schmidt (1997) and Shea et al. (2001) claimed that learning in continuous-tracking tasks occurred independently of the conscious knowledge of repetitions. This claim is at odds with the conclusion reached with SRT tasks and other implicit-learning situations. In a prior comment paper (Perruchet, Chambaron, \& Ferrel-Chapus, 2003), we tentatively attributed this discrepancy to the measure of conscious knowledge in the Wulf and collaborators studies. This hypothesis is not invalidated in the present experimental contribution. It may apply, for instance, to Wulf and Schmidt (1997, Exp. 2), in which the possibility of learning cannot be ruled out. However, by and large, it appears that the conclusions of Wulf and

\footnotetext{
${ }^{2}$ This conclusion is compatible with the earlier results of Pew (1974, Exp. 1). Pew did not provide sufficient methodological details to assess whether the criticisms directed towards the Wulf and collaborators studies also apply to his experiment. It is not even clear whether the repeated segment differed for each participant or not. However, even if one takes the evidence provided in Pew's experiment at face value, it is worth emphasizing that learning required quite extensive practice. Participants tracked the target for 1 hour each day for 16 days, and the difference in performance between the random and the repeated segments reached significance only from Day 6 onward.
} 
collaborators regarding the dissociation between performance and explicit knowledge may receive a simpler explanation. If there is no reliable evidence for an improvement in motor performances on the repeated segment, then the lack of explicit knowledge about this segment becomes a trivial outcome.

Our reappraisal has also other, more fundamental, implications. Some early publications have favoured a view according to which implicit-learning mechanisms are ubiquitous, allowing behavioural adaptation whatever the degree of complexity of the situation with which the learner is faced. The well-known difficulty of publishing null results or replication failures has contributed to maintenance of this view. This state of affairs is prejudicial to our understanding of the processes involved in implicit learning. The possibility of clearly delineating the situations in which learning is possible from those in which learning is not possible offers a valuable constraint for theoretical speculations. In this context, the present study opens the following challenge. In spite of a close parallel between continuoustracking tasks and SRT tasks, taking benefit from the repetition of a segment in continuoustracking tasks appears to be considerably more difficult than taking benefit from the repetition of a sequence in SRT tasks. How can this discrepancy be explained?

Pending further empirical and theoretical studies, we are limited to some speculative hypotheses. A possible scenario is the following. There is now good evidence that the improved performance of participants in the SRT tasks is due to the increased sensitivity of participants to the frequency of certain chunks (typically short fragments of two or three trials; e.g., Buchner, Steffens, \& Rothkegel, 1998; Perruchet \& Amorim, 1992). There is no a priori reason not to apply a similar model to any task. Indeed, the formation of chunks is thought of as dependent on fundamental constraints of human attentional processing and thus is not limited to a specific format of input. As a consequence, it can be assumed with reasonable confidence that any participant dealing with a continuous-tracking task also codes the incoming information as a succession of chunks. However, forming chunks is not a guarantee for learning by itself. In order to promote learning, the chunks formed by the learner must have a chance of being repeated. The difficulty of learning from continuoustracking tasks could stem from the fact that a continuous displacement does not provide compelling cut-offs to serve as chunk boundaries. If a participant tracks a target following a fixed pattern several times, while chunking this pattern into different units on each time, then there is no way to detect the repetition structure, because each chunk will be unique.

The above analysis is open to empirical investigation, and other interpretations are certainly possible. Irrespective of the final outcome of further investigations, the reported difficulty in profiting from repetitions in continuous-tracking tasks is challenging and thought provoking, given the close similarity between these tasks and some other conventional situations of implicit learning. Finding the key difference responsible for this empirical discrepancy will certainly be fruitful for our understanding of implicit-learning mechanisms.

Original manuscript received 16 September 2004 Accepted revision received 11 May 2005 PrEview proof published online 20 September 2005

\section{REFERENCES}

Buchner, A., Steffens, M., \& Rothkegel, R. (1998). On the role of fragmentary knowledge in a sequence learning task. Quarterly Journal of Experimental Psychology, 51A(2), 251-281.

Cleeremans, A., Destrebecqz, A., \& Boyer, M. (1998). Implicit learning: News from the front. Trends in Cognitive Sciences, 2, 406-416.

Curran, T. (1997). Effects of aging on implicit sequence learning: Accounting for sequence structure and explicit knowledge. Psychological Research, 60, 24-41.

Kelly, S. W., Burton, A. M., Riedel, B., \& Lynch, E. (2003). Sequence learning by action and observation: Evidence for separate mechanisms. British Journal of Psychology, 94, 355-372. 
Meulemans, T., Van Der Linden, M., \& Perruchet, P. (1998). Implicit sequence learning in children. Journal of Experimental Child Psychology, 69, 199-221.

Perruchet, P., \& Amorim, M. A. (1992). Conscious knowledge and changes in performance in sequence learning: Evidence against dissociation. Journal of Experimental Psychology: Learning, Memory, and Cognition, 18, 785-800.

Perruchet, P., Chambaron, S., \& Ferrel-Chapus, C. (2003). Learning from implicit learning literature: Comment on Shea, Wulf, Whitacre, and Park (2001). Quarterly Journal of Experimental Psychology, 56A, 769-778.

Pew, R. W. (1974). Levels of analysis in motor control. Brain Research, 71, 393-400.

Shanks, D. R., \& Perruchet, P. (2002). Dissociation between priming and recognition in the expression of sequential knowledge. Psychonomic Bulletin and Review, 9, 362-367.

Shea, C. H., Wulf, G., Whitacre, C. A., \& Park, J.-H. (2001). Surfing the implicit wave. Quarterly Journal of Experimental Psychology, 54A, 841-862.

Smith, J., Siegert, R., McDowall, J., \& Abernethy, D. (2001). Preserved implicit learning on both the serial reaction time task and artificial grammar in patients with Parkinson's disease. Brain and Cognition, 45, 378-391.

Wulf, G., \& Schmidt, R. A. (1997). Variability of practice and implicit motor learning. Journal of Experimental Psychology: Learning, Memory, and Cognition, 23, 987-1006.

Wulf, G., \& Shea, C. H. (2002). Principles derived from the study of simple skills do not generalize to complex skill learning. Psychonomic Bulletin and Review, 9, 185-211. 